\title{
T-cell predominant balanitis in a traumatic tetraplegic patient: a case report
}

\author{
D Van Velzen ${ }^{2}$, S Vaidyanathan ${ }^{1}$, KR Krishnan ${ }^{1}$, BM Soni $^{1}$ and KF Parsons ${ }^{1}$ \\ ${ }^{1}$ Regional Spinal Injuries Centre, Southport, Merseyside, UK; ${ }^{2}$ Department of Fetal and Infant Pathology, University \\ of Liverpool, Liverpool, $U K$
}

\begin{abstract}
T-cell predominant balanitis is described in a 38 year old uncircumcised tetraplegic man whose presenting feature was non-progressive red lesions over the inner prepuce and the glans penis without signs of infection, phimosis, or meatal stenosis. There was no regional lymphadenopathy. He was not exposed to any of the high risk factors for human immunodeficiency virus infection. As the lesion did not respond to topical antibacterial, antifungal, and corticosteroid medications applied in that order, circumcision was performed. Following circumcision, the remaining lesion over the glans penis regressed completely over a period of 1 month. Histopathology of the excised prepuce revealed both areas of normal nonkeratinizing squamous epithelium as well as areas with hyperplasia. No atypia was noted and Bowenoid changes were not seen. Immunohistochemical studies on the inflammatory infiltrate in the excised prepucial lesion using tissue proliferation markers (PCNA and MIB-1) revealed active proliferation of the band-like infiltrate shown by immunophenotyping (anti-human $\mathrm{T}$ cell, CD45RO, clone UCHL1 and L26, pan-B marker) to consist predominantly of T-cells, further supporting the hypothesis of a local immune-mediated inflammatory process as the final pathogenic mechanism of the penile lesion.
\end{abstract}

Keywords: balanitis; T-cell; spinal cord injury

\section{Introduction}

Penile lesions in spinal cord injury patients are rare, the commonest being balanoposthitis of infective aetiology (bacterial or fungal) seen in those wearing a penile condom for urinary drainage especially in those with stasis of urine in the condom. Other uncommon lesions are (i) a pressure sore on the undersurface of the glans penis in those patients with a long penis and a small scrotum; (ii) traumatic lesions of the glans penis when a paraplegic/tetraplegic person who is on indwelling catheter drainage, performs sexual intercourse after strapping the catheter to the undersurface of the flaccid penis. When the penis becomes erect, the catheter tends to cut through the inferior aspect of the urethral meatus. As spinal cord injury patients use a variety of skin care products for personal hygiene as well as to soothe, smooth, and hydrate their neuropathic skin, they are prone to devlop a 'reaction' to any of these products; precise identification of the offending substance may be difficult. We recently observed a non-progressive, reddish lesion over the glans penis and inner prepuce in a tetraplegic patient, not responsive to topical antibacterial, antifungal, and

Correspondence: S Vaidyanathan, Regional Spinal Injuries Centre, District General Hospital, Town Lane, Kew, Southport, Merseyside PR8 6PN, UK corticosteroid treatment. Circumcision biopsy was performed to exclude any pre-malignant lesion. Double immuno-histochemical staining with pan $T$ cell/B-cell markers and proliferation markers (PCNA/ MIB-1) showed a band-like infiltrate with dense continuous staining of the vast majority of cells with a monoclonal antibody anti-human $\mathrm{T}$ cell, CD45RO, clone UCHL1 and amongst these infiltrates, a relatively high fraction staining positive for cell proliferation markers, thus indicating extensive commitment to S-phase. The rarity of this type of clinical and immunohistochemical picture prompted us to report this case.

\section{Case report}

The patient sustained a fracture of C- 6 with complete C-6/C-7 tetraplegia in a car accident in 1977 when he was 20 years old. He underwent repair of a left ischial pressure sore in 1992. He was on penile condom drainage until July 1993 when a residual urine of $750 \mathrm{ml}$ was noted. Since then he has been on indwelling urethral catheter drainage. In August 1994, he noted erythematous areas over the glans penis and inner prepuce (Figure 1). There was no induration, or rubbery feeling, or pustules, or ulceration, or bleeding, or meatal stenosis and there was no phimosis. The 


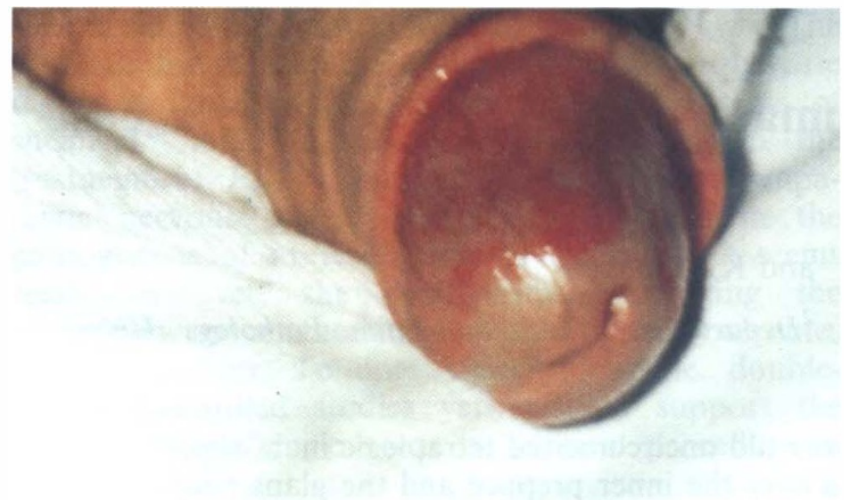

follicle centre reaction or mantle zone (Figure 3), at a level somewhat below that of the band of $\mathrm{T}$-cells which was found directly opposed to either normal or hyperplastic squamous epithelium or was found separated from the, usually hyperplastic, epithelium by a zone of homogenously staining, hyalinised eosinophilic ground substance.

Proliferation assessment was made using commercially available monoclonal antibodies against markers (PCNA/PC10 DAKOPATS and MIB-1 (cDNA defined Ki-67 antigen segment) suitable for use in routinely formalin fixed and paraffin embedded material. For PCNA staining, the primary antibody (PCNA 10, DAKO Product number 0505) was used in a 1:300 dilution; the MIB-1/Ki-67 equivalent antigen antibody (DAKO Product number M79) was used in a

Figure 1 Photograph of the penis of a 37 year old traumatic tetraplegic showing the erythematous area over the glans penis and the prepuce.

prepuce was soft, and thin, and could be retracted without any difficulty. The uninvolved portion on the inner aspect of the prepuce and the exterior of the prepuce was of normal appearance and texture. There was no inguinal lymphadenopathy. He was not exposed to any of the high risk factors for human immunodeficiency virus infection. The lesions over the glans penis and prepuce were unresponsive to topical antibacterial, antifungal and corticosteroid therapy applied in that temporal sequence for a fortnight each as the initial clinical diagnosis was either bacterial or fungal infection. The serum immunoglobulin profile was normal (IgG: $8.84 \mathrm{~g} / \mathrm{l} ; \operatorname{IgA}: 3.31 \mathrm{~g} / \mathrm{l} ; \operatorname{IgM}: 1.14 \mathrm{~g} /$ 1). Over a period of 6 months of observation, the lesions were not found to be progressive. As he was anxious that the reddish lesion would not prove to be a malignant or a pre-malignant condition, circumcision was performed in February 1995. Following circumcision, the reddish lesions on the glans penis regressed completely over a period of 1 month.

Histopathology of the excised prepuce revealed both areas of normal non-keratinizing squamous epithelium as well as areas with hyperplasia. No atypia was noted and Bowenoid changes were not seen. Focally, a more atrophic thin lining was seen with complete loss of rete ridges; and occasional clefting of the squamous epithelial layer from the supporting basement membrane was noted. A band-like inflammatory infiltrate was noted consisting mainly of $\mathrm{T}$ cells (Figure 2) as defined by immunohistochemical staining using commercially available monoclonal mouse anti-human $\mathrm{T}$ cell, CD45RO clone UCHL1 (which recognises a $180000 \mathrm{MW}$ component of the human leucocytecommon antigen, CD45, DAKO) and L26 (Pan-B marker, DAKO) suitable for use on formaldehyde fixed and routinely paraffin embedded tisse. B-cells were only found in small follicular aggregates without

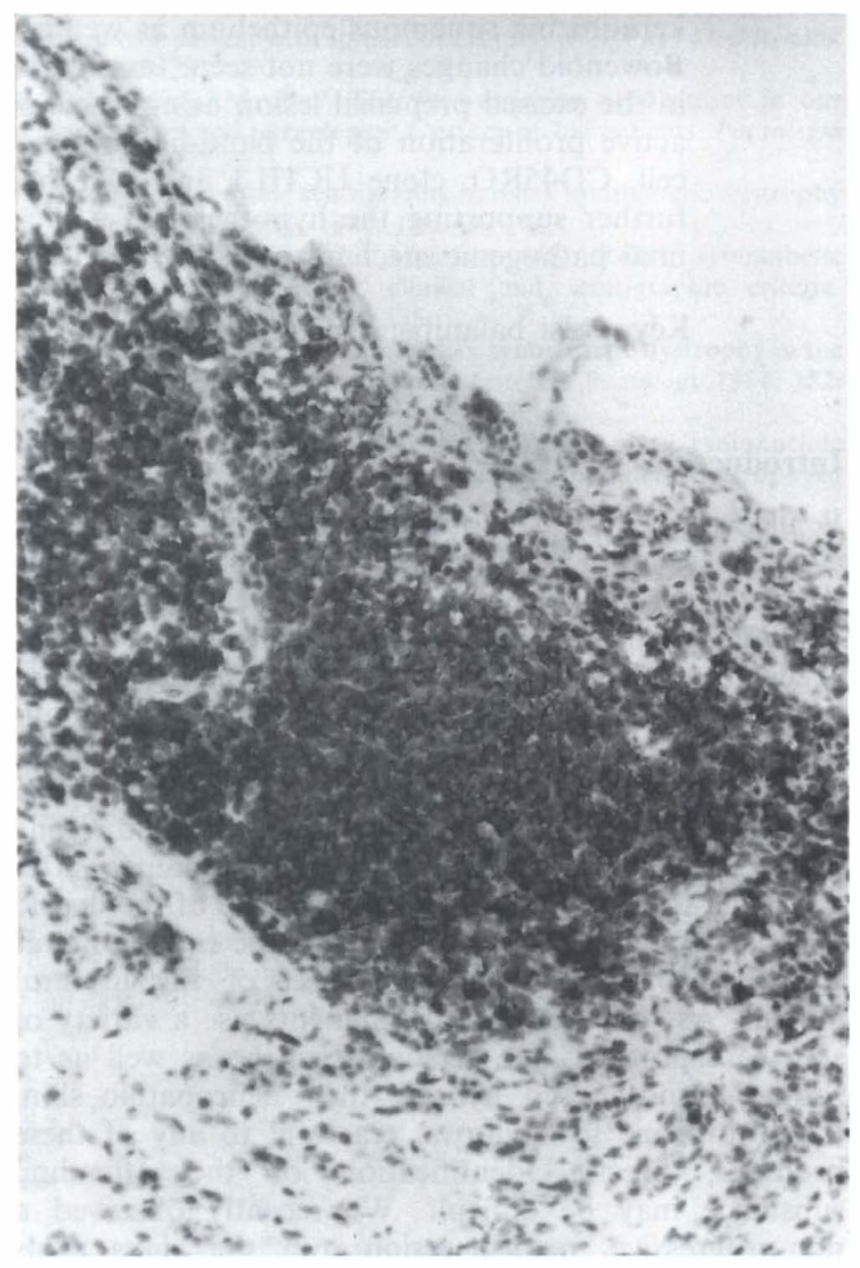

Figure 2 Microphotograph of partially denuded preputial tissue showing band like infiltrate stained with monoclonal mouse anti-human T cell, CD45RO clone UCHL1 (which recognises a $180000 \mathrm{MW}$ component of the human leucocytecommon antigen, CD45, DAKO). Note dense continuous staining of vast majority of cells. 5 Micron paraffin section, microscopical magnification $40 \times$ 


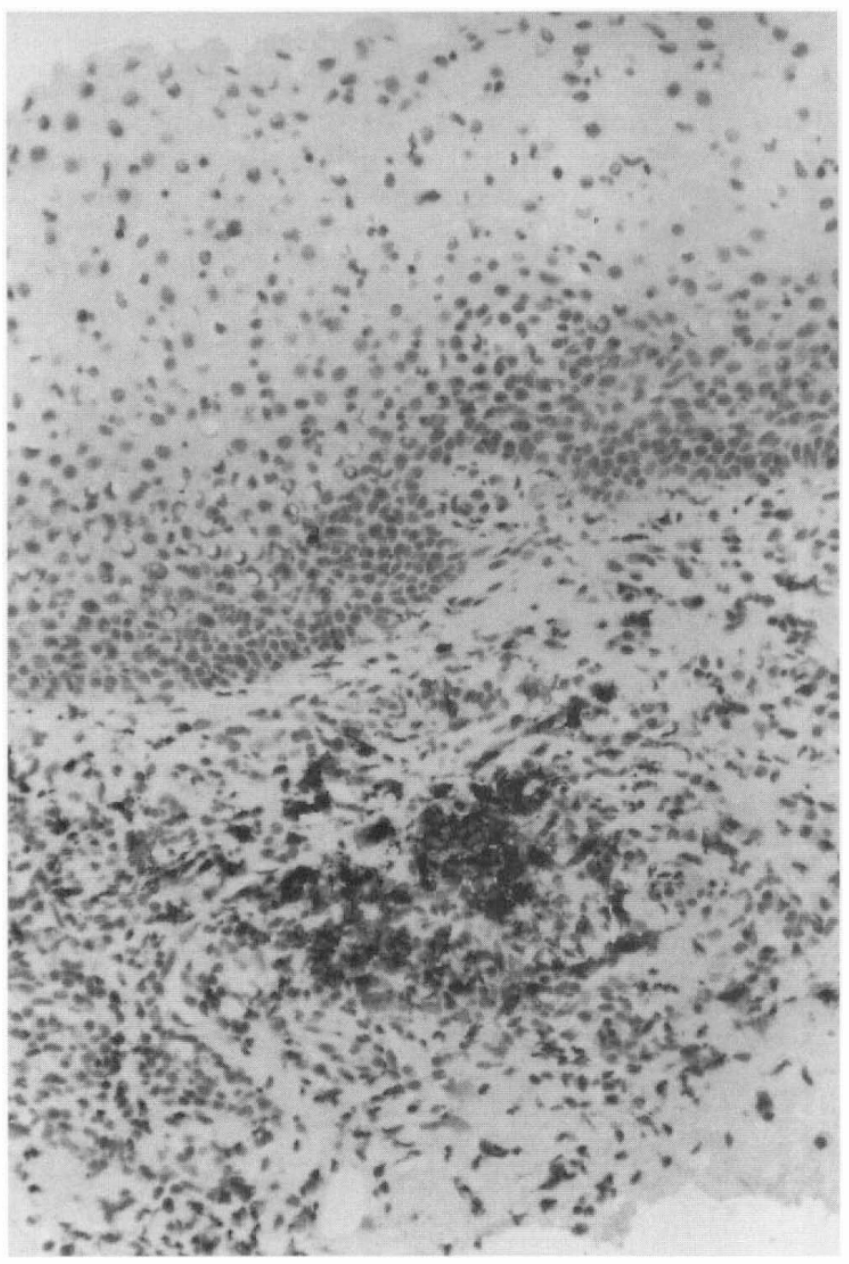

Figure 3 Microphotograph of non-denuded section of preputial resection specimen featuring one of rare, relatively deeply positioned aggregates stained with L26 (Pan-B marker, DAKO). Note small aggregate of positive cells surrounded by large numbers of cells not recognised as of Bcell phenotype. 5 Micron paraffin section, microscopical magnification $40 \times$

$1: 200$ dilution. This showed high proliferation rates of T-cells (PCNA 40-60\%, MIB-1 15-30\%) with virtually absent proliferation of any B-cells.

\section{Discussion}

The differential diagnosis of the reddish lesion over the prepuce and glans penis include (i) Zoon's balanitis, or plasma cell balanitis, (ii) candidal balanitis, (iii) Carcinoma-in-situ (erythroplasia of Queyrat) and, (iv) contact dermatitis. Zoon's balanitis is characterised by an extensive infiltration of plasma cells without evidence of dysplasia of the overlying epidermis. The classical clinical picture of plasma cell balanitis consists of a shiny, glazed red macular erythematous lesion with multiple pinpoint, bright red spots ('cayenne pepper spots'). Immunohistologically, $\operatorname{IgE}$ and $\operatorname{IgG}$ are found to be dominant immunoglobulin classes in the plasma cell infiltrate. ${ }^{1}$ The aetiology of Zoon's balanitis is not clearly established. Chronic infection by Mycobacterium smegmatis has been postulated; heat, constant friction and poor hygiene may be predisposing factors. ${ }^{2}$ Immunofluorescence studies using FITCconjugated IgG fraction goat antibodies directed against human $\operatorname{IgG}, \operatorname{IgA}, \operatorname{IgM}, \operatorname{IgD}$ and $\operatorname{IgE}$ showed that IgE-positive cells appeared to be the most frequent in the plasma cell infiltrate. ${ }^{1}$ However, in this case, double immunohistochemical staining showed the inflammatory infiltrate to be mainly of T-cells, with high proliferation rates of T-cells (PCNA 40-60\%, MIB-1 15-30\%) and virtually absent proliferation of any B-cells, thus ruling out the diagnosis of Zoon's balanitis.

Hinchliffe et al. ${ }^{3}$ observed a band-like infiltrate of more or less pure $\mathrm{T}$ cell nature in all 20 paediatric patients diagnosed clinically as balanitis xerotica obliterans/lichen sclerosus et atrophicus in the paediatric urology department of a children's hospital. But neither the classical clinical picture of mature balanitis xerotica obliterans viz. sharply marginated white patch on the glans penis that often surrounds or even involves the urethral meatus, and a scar-like feeling (sclerosis) on palpation, nor other less frequently observed clinical symptomatology such as recurrent balanoposthitis, oedematous foreskin, tight, non-retractable foreskin, meatal stenosis, etc. was present in this case. Therefore, we preferred to label this case as 'T-cell predominant balanitis'. The observed predominantly T-cell response in this patient might represent dermal hypersensitivity reaction to Mycobacterium smegmatis, or to any of the ingredients of skin care products applied over the penis. As spinal cord injury patients use a variety of skin care products for personal hygiene as well as to sooth, smooth, and hydrate their neuropathic skin, they are prone to develop a 'reaction' to any of these products; precise identification of the offending substance may be difficult, if not impossible. As this lesion developed while the patient was on indwelling catheter drainage, dermal reaction to a condom may be excluded. Dermal hypersensitivity reaction to smegma bacillus would explain the gradual disappearance of the remaining lesion after cirumcision as after circumcision, the glans penis was no longer exposed to accumulation of smegma in the prepucial sac. It may be interesting to study lymphocyte surface antigen abnormalities in cases of dermal hypersensitivity reaction with no known malignant potential (for example, this patient), as lymphocyte surface markers can rarely be altered in benign reactive conditions. ${ }^{4}$

The presence of red, recalcitrant penile lesions, is often disturbing to the patient and more so in a person with traumatic tetraplegia and to his partner, as they are more vulnerable to stressful events in life after having gone through a period of severe stress reaction during the initial episode of cervical spinal injury. It becomes imperative to confirm or exclude a premalig- 
nant lesion of the penis not only to allay the anxiety of the patient and his carer(s) but also to institute appropriate surgical treatment in case of precancerous change. Furthermore, accurate diagnosis will allow for the cessation of various treatment modalities such as topical antibiotics, topical antifungal, or topical corticosteroids which may not be indicated, or to empirically change the skin care product which the patient has been applying over the penis in case the skin lesion is one of dermal hypersensitivity reaction to an environmental factor. As differential diagnosis on clinical symptomatology was not possible, we decided to perform circumcision in this patient with traumatic tetraplegia with a reddish lesion over the glans penis and the prepuce. Circumcision is a simple procedure to carry out, and it would provide sufficient material for exact hisopathological diagnosis of the penile lesion which will also allow discussion of long term prognosis with the patient and his partner.

The treatment of a penile lesion is often initiated by the patient either because of symptoms such as itching, or bleeding, or because of ghastly appearance in a sensitive area of the body. Thus, for the spinal cord injury patient with a penile lesion in whom the subjective feeling of discomfort or pain does not provide the stimulus to seek prompt therapy, the risk exists of a delay in seeking medical help especially if the lesion should prove to be a precancerous or overtly malignant condition. It is the role of the attentive carer or clinician to detect such lesions in an early stage and initiate appropriate medical treatment without undue delay. Awareness of the occurrence of penile lesions in the spinal cord injury patients who run the risk of poor or limited penile hygiene (especially in those who wear a penile condom for urinary drainage), will help towards early recognition of these conditions, and any inciting factors for chronic irritation may be removed without delay.

\section{References}

1 Nishimura M, Matsuda T, Muto M, Hori Y. Balanitis of Zoon. International Journal of Dermatology 1990; 29: 421 - 423.

2 Jolly BB, Krishnamurty S, Vaidyanathan S. Zoon's balanitis. Urologia Internationalis 1993; 50: $182-184$

3 Hinchliffe SA, et al. Composition of the inflammatory infiltrate in paediatric penile lichen sclerosus et atrophicus (Balanitis Xerotica Obliterans): A prospective, comparative immunophenotyping study. Paediatric Pathology 1994; 14: 223-233.

4 Smoller BR, et al. Lymphocyte antigen abnormalities in inflammatory dermatoses. Applied Immunohistochemistry 1995; 3: $127-131$. 